\title{
What happened before the Big Bang?
}

\author{
MARTIN BOJOWALD \\ Institute for Gravitational Physics and Geometry, The Pennsylvania State University, 104 Davey Laboratory, University Park, Pennsylvania 16802, USA \\ e-mail: bojowald@gravity.psu.edu
}

Published online: 1 July 2007; doi:10.1038/nphys654

Was the Universe before the Big Bang of classical nature, described well by a smooth space-time? Or was it in a highly fluctuating quantum state? This is one of the most basic questions that we may ask once it is accepted that there was something before the Big Bang. Loop quantum gravity ${ }^{1-3}$ applied to isotropic models ${ }^{4}$ has shown that the quantum evolution of a wavefunction extends through the Big Bang ${ }^{5}$. Although a general demonstration is still lacking, this may suggest that calculations, and possibly future indirect observations, may allow us to see the Universe as it was before the Big Bang. Here, we analyse an explicit model with a pre-Big Bang era, indicating limitations that would imply that it is practically impossible to answer some of our questions. Assumptions (or prejudice) will remain necessary for knowing the precise state of the Universe, which cannot be fully justified within science itself.

Even with deterministic evolution for cosmological quantum systems being valid at all times, there may remain limitations to our knowledge of the Universe phases before the Big Bang. To analyse this, we use a mathematical model that is exactly solvable ${ }^{6}$ and clearly shows all details of its behaviour. (Earlier numerical studies $^{7}$ did not reveal the subtle properties derived here from exact solutions.) Solvable models have often played a central role in understanding detailed properties in a new field, and we can expect this to also be true for quantum cosmology. Due care must be exercised when using such models that may have special properties not extending to realistic situations. But a solvable model shows a minimum of the complexity that must be expected for realistic situations. Thus, in contrast to detailed quantitative calculations, a proof of the inability to obtain certain precise predictions in a solvable model can be taken as a more reliable result.

In this context, it is often taken for granted that the existence of a bounce in cosmology, that is, a volume bounded away from zero during evolution, makes it possible to extrapolate all physical quantities to times before the Big Bang. As we will see here, this expectation is not even realized in a solvable model of minimum computational complexity. A memory of certain aspects of the Universe before the Big Bang, chiefly related to fluctuations relevant for the basic question posed here, is lost while transiting through the Big Bang. This is not simply saying that the Universe was in a highly quantum phase at the Big Bang, thus shrouding its pre-history by quantum uncertainty. As we will be dealing with a fully quantized system and its degrees of freedom, we could in principle calculate backwards the state before the Big Bang. As we will see, on the basis of precise analytical solutions for fluctuations evolving through a bounce, the new aspect of quantum cosmology is that such an extrapolation would require exceedingly precise knowledge of the present state that cannot realistically be obtained. Nevertheless, in this model it can precisely be answered what aspects of a pre-Big Bang universe can be determined and to what degree. It thus exhibits predictivity, even in its inability to make certain predictions.

Our model is spatially isotropic everywhere, that is, its properties are identical in different spatial positions as well as in different directions. There is a single matter component that is massless and does not have self-interactions. Interactions between matter and gravity are, however, fully included and crucial. Classical evolution equations of the system follow from Einstein's equation of general relativity. For the model considered here, it reduces to the Friedmann equation $a^{-2}(\mathrm{~d} a / \mathrm{d} t)^{2}=4 \pi G p_{\phi}^{2} / 3 a^{6}$ for the scale factor, $a$, of the Universe as a function of coordinate time, $t$. The rate of change of $a$ is determined by the momentum $p_{\phi}$ conjugate to the matter component, $\phi$, coupled through the gravitational constant, G. A canonical analysis shows that $q$, defined as $\mathrm{d} a / \mathrm{d} t$, can be used as the gravitational configuration variable with momentum $p=a^{2}$. Solving for $p_{\phi}$ then implies $\left|p_{\phi}\right|=|H|$, where $H=q p$ in appropriate units absorbing numerical pre-factors. This equation means that $H$ can be used as a hamiltonian determining evolution by $\mathrm{d} q / \mathrm{d} \phi=\partial H / \partial p=q$ and $\mathrm{d} p / \mathrm{d} \phi=-\partial H / \partial q=-p$. These are evolution equations for the gravitational variables with respect to $\phi$ rather than a coordinate time that can be eliminated in generally covariant systems. They are easily solved completely by $q(\phi)=q_{0} \mathrm{e}^{\phi}$ and $p(\phi)=p_{0} \mathrm{e}^{-\phi}$. (Despite the formal resemblance, this is not a solution for static de Sitter space as $q$ and $p$ are expressed in terms of $\phi$ rather than coordinate time, $t$.) The Big Bang singularity is shown in the solutions as a point where $p$, related to the spatial volume by $V \propto p^{3 / 2}$, approaches zero and the matter field, $\phi$, as well as the extrinsic curvature, $q$, becomes infinite. A space-time analysis shows that this point would be reached in a finite amount of proper time for any observer.

In general, it is highly non-trivial for a classical solvable system to remain solvable after quantization. A quantum state contains many more parameters than a classical state, as not only $q$ and $p$ must be determined as mean values $\langle\hat{q}\rangle_{\psi}$ and $\langle\hat{p}\rangle_{\psi}$ in a state $\psi$, but also fluctuations

$$
\Delta q=\sqrt{\left\langle\hat{q}^{2}\right\rangle_{\psi}-\langle\hat{q}\rangle_{\psi}^{2}}, \quad \Delta p=\sqrt{\left\langle\hat{p}^{2}\right\rangle_{\psi}-\langle\hat{p}\rangle_{\psi}^{2}}
$$

and infinitely many more quantities involving higher powers of $\hat{q}$ and $\hat{p}$. These are new ingredients due to the probabilistic nature of quantum theory. The fluctuation, $\Delta q$, for example, gives the fundamental variance in repeated measurements of $q$. In quantum cosmology of a single universe, such an operational interpretation of $\Delta q$ is conceptually difficult, but for our purposes it is sufficient to regard $\Delta q$ as a dynamical measure of quantum aspects of the state. In fact, quantum mechanics provides the dynamical evolution of all fluctuations and other variables: a semiclassical wavepacket spreads and deforms when it evolves (changing $\Delta q$ and so on), 


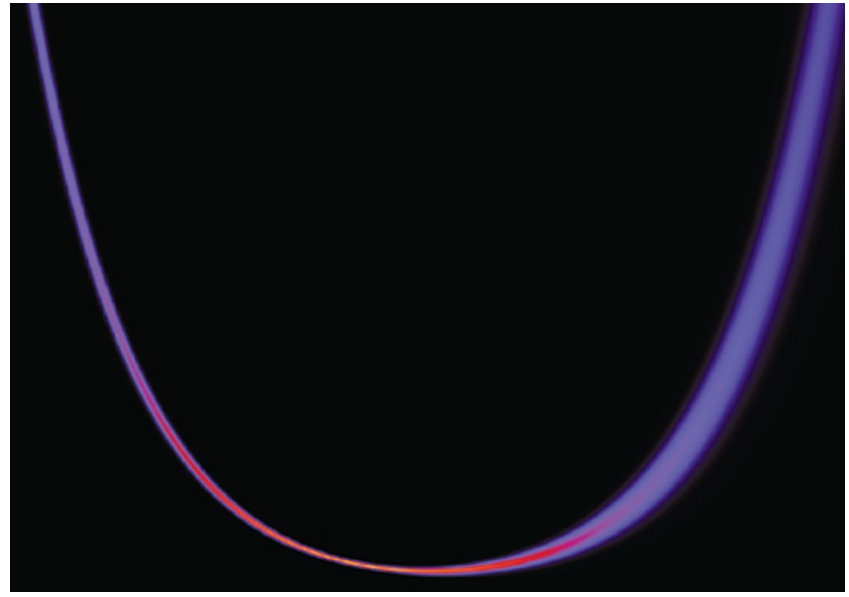

Figure 1 Spreading through a bounce. A state that initially has small fluctuations (left) bounces and develops larger fluctuations (right). Time proceeds along the horizontal axis, with the volume plotted vertically. The magnitude of the wavefunction is indicated by colours. Yellow signifies the highest amplitude realized where the state is sharpest, followed by red and blue.

which in turn affects the motion of its mean at $\langle\hat{q}\rangle$ and $\langle\hat{p}\rangle$. This back-reaction implies quantum corrections to the classical equations that can often be observed directly. The coupled dynamics is usually complicated, but for quadratic hamiltonians, such as our system, a detailed analysis becomes possible even in quantum theory. We have Heisenberg-type equations $\mathrm{d}\langle\hat{q}\rangle / \mathrm{d} \phi=\langle[\hat{q}, \hat{H}]\rangle / i \hbar=\langle\hat{q}\rangle$ and $\mathrm{d}\langle\hat{p}\rangle / \mathrm{d} \phi=\langle[\hat{p}, \hat{H}]\rangle / i \hbar=-\langle\hat{p}\rangle$, where, for example, $[\hat{q}, \hat{H}]=\hat{q} \hat{H}-\hat{H} \hat{q}$, for expectation values. In addition, there are equations of motion for fluctuations such as $\mathrm{d}(\Delta p)^{2} / \mathrm{d} \phi=\left\langle\left[\hat{p}^{2}, \hat{H}\right]\right\rangle / i \hbar-2\langle\hat{p}\rangle\langle[\hat{p}, \hat{H}]\rangle / i \hbar=-2(\Delta p)^{2}$ that can also be computed and solved easily. These equations contain the new information of a quantum system compared with a classical one. They tell us not only how mean values change in time but also how the expected deviation of an actual measurement from the expected value evolves. If this variance is large, we have typical quantum behaviour.

Importantly, a solvable system is thus available for cosmology. It allows us to include perturbations, which, around the harmonic oscillator, is the basis of much of the phenomenology of particle physics. With the solvable cosmological model identified here, a similar systematic perturbation theory ${ }^{8}$ becomes available for quantum cosmology. However, there is one further step to obtain a non-singular system, reaching before the Big Bang. Quantum gravity effects at high curvature, $q$, change the hamiltonian, in a loop quantization to

$$
\hat{H}=\widehat{\sin (q)} \hat{p},
$$

which agrees with the one above only for small curvature, $q \ll 1$, but differs at high curvature close to the Big Bang. Although this hamiltonian is not quadratic in $q$ and $p$ and thus not obviously solvable, the quantum system is, remarkably, still solvable. Introducing the complex variable $\hat{J}=\hat{p} \mathrm{e}^{\hat{i q}}$, the hamiltonian

$$
\hat{H}=(\hat{J}-\hat{\bar{J}}) / 2 i
$$

becomes linear and gives $(1 / 2 i)\left(\mathrm{e}^{i q}-\mathrm{e}^{-i q}\right) p=\sin (q) p$ as the correct classical correspondence. With a nonlinear expression, $J$, in $q$ and $p$ this does not immediately imply solvability, but all evolution equations for expectation values

$$
\mathrm{d}\langle\hat{p}\rangle / \mathrm{d} \phi=\langle[\hat{p}, \hat{H}]\rangle / i \hbar=-(1 / 2)(\langle\hat{J}\rangle+\overline{\langle\hat{J}\rangle})
$$

and $\mathrm{d}\langle\hat{J}\rangle / \mathrm{d} \phi=\langle[\hat{J}, \hat{H}]\rangle / i \hbar=-\langle\hat{p}\rangle$ as well as fluctuations can, as before, not only be computed but even be solved explicitly in general form. Once reality conditions are implemented to ensure that $q$ contained in $J$ is real $\left(|J|^{2}=p^{2}\right)$, the solution for $p$ is given by a function $p(\phi)=H \cosh (\phi)$ that never becomes zero. Thus, the solution is free of singularities where space would collapse to a single point. There is a minimum extension instead, which is the bounce replacing the Big Bang singularity. All variables evolve deterministically through the bounce, including quantum fluctuations (Fig. 1).

We can now formulate our basic question about fluctuations before and after the Big Bang. A state can be considered to be more classical the smaller the quantum fluctuations are, with a lower limit given by Heisenberg's uncertainty relation $(\Delta q)^{2}(\Delta p)^{2} \geq \hbar^{2} / 4$. This can at best be saturated, which we can safely assume to model our present Universe. We ask whether this observation of present classical behaviour would put any restrictions on fluctuations before the Big Bang. Proceeding as before ${ }^{9}$, fluctuations depend on three free constants $\Delta_{ \pm}, \Delta_{0}: \Delta p(\phi)^{2}=\Delta_{-}^{2} \mathrm{e}^{-2 \phi}+\Delta_{0}^{2}+\Delta_{+}^{2} \mathrm{e}^{2 \phi}$ where $\Delta_{0}$ is relevant only near the bounce $(\phi=0)$ and must be small for matter not to have strong fluctuations. The remaining parameters are volume fluctuations before $\left(\Delta_{-}\right)$and after $\left(\Delta_{+}\right)$the Big Bang. They appear in the uncertainty product, which is easier, and equally informative, to write between $\Delta p$ and $\Delta H$ :

$$
\begin{aligned}
(\Delta H)^{2}(\Delta p)^{2} & =(\Delta H)^{2}\left(\Delta_{-}^{2} \mathrm{e}^{-2 \phi}+\Delta_{0}^{2}+\Delta_{+}^{2} \mathrm{e}^{2 \phi}\right) \\
& \geq \hbar^{2}\left(p(\phi)^{2}-H^{2}\right) / 4 .
\end{aligned}
$$

At large $\phi>0$, the constant $\Delta_{-}$is negligible (our present Universe, for comparison, would at least have $\left.\mathrm{e}^{\phi_{0}} \approx p\left(\phi_{0}\right) / p(0) \approx \mathrm{e}^{120}\right)$. Semiclassicality today requires $\Delta_{+}$to be about $\hbar H / \Delta H$, whereas $\Delta_{-}$does not feature at all and is observationally unrestricted, being suppressed by a factor $\mathrm{e}^{-4 \phi}$ compared with $\Delta_{+}$. It does, however, become the essential quantity before the Big Bang $(\phi<0)$. We would have small fluctuations at that time if $\Delta_{-}$is also of the order of $\hbar H / \Delta H$, which is certainly allowed as a solution. But equation (1), or other available information on $\Delta p(\phi)$ at $\phi>0$, does not restrict $\Delta_{-}$; it can be huge (as long as it is smaller than $\mathrm{e}^{4 \phi_{0}} \approx \mathrm{e}^{480} \approx 10^{200}$ ) and imply strong quantum fluctuations throughout the pre-Big Bang Universe. It is practically impossible to draw conclusions about fluctuations of the Universe before the Big Bang: the relevant parameter is suppressed by the sheer size of the present Universe. (There are physical processes called decoherence $^{10}$ that can push a quantum system to more classical behaviour. These complicated mechanisms depend on the quantum state on which they work, and especially fluctuations then remain important for the outcome.)

This rather simple derivation illustrates limitations to our understanding of nature. Several examples, in chaotic dynamics and quantum theory, are known, all having played important roles in philosophical debates. We should therefore distinguish this from the limitation arising here, which is of a different type. The system is not chaotic, but any trace of some of its initial conditions is lost in time. It is a quantum system and the limitation is related to quantum uncertainties. As is usual, generic states spread and their fluctuations grow. But in cosmology, fluctuations before and after the Big Bang are largely independent; knowledge of fluctuations themselves is limited in addition to the uncertainty of 
mean values implied by non-zero fluctuations. In cosmology, there is an immense range of scales involved from the Planck length to the Hubble radius. Our detailed analysis shows how they can enter predictions in unexpected ways.

Physics has been leading to observations and an understanding of ever earlier times of our Universe and possibly even its origin, but the Big Bang always occurred as a barrier. Even in models whose mathematical equations do not break down, describing a universe before the Big Bang, some information about one of the most elementary properties is practically lost. Specifically, in the solvable model considered here, the size of quantum fluctuations before the Big Bang enters post-Big Bang quantities only in a highly suppressed way. This suggests that current observations cannot give significant constraints on their values.

As a general concept, this presents an attractive new universe picture, combining perpetual cyclic models and linear ones. A linear model starts at a finite time in the past, whereas a cyclic model exists forever, be it for several cycles or only for one contracting phase followed by an expanding one. We described a theory whose evolution never stops, being cyclic in this sense. But some traces of each cycle are irretrievably lost shortly after transiting from collapse to expansion. Complete predictions and explanations of observations can only be made for the finite part starting after the Big Bang. An eternal recurrence of the same is prevented by intrinsic cosmic forgetfulness. This may seem like a return to the traditional Big Bang picture, where speaking of 'before the Big Bang' is meaningless. But it is more subtle: in this traditional picture, the Big Bang is preceded by a singularity where the theory breaks down. The singularity is a theoretical limitation, rather than a physical beginning. Quantum gravity as used here can provide solutions extending through the classical singularity. And yet, limitations to observations of some, but not all, pre-Big Bang properties exist that are now derived within the theory, not as limitations to the theory.

Received 12 March 2007; accepted 4 June 2007; published 1 July 2007.

References

1. Rovelli, C. Quantum Gravity (Cambridge Univ. Press, Cambridge, 2004).

2. Ashtekar, A. \& Lewandowski, J. Background independent quantum gravity: A status report. Class. Quantum Grav. 21, R53-R152 (2004)

3. Thiemann, T. Introduction to modern canonical quantum general relativity. Preprint at $<$ http://arxiv.org/abs/gr-qc/0110034> (2001).

4. Bojowald, M. Loop quantum cosmology. Living Rev. Relativity 8, 11 (2005).

5. Bojowald, M. Absence of a singularity in loop quantum cosmology. Phys. Rev. Lett. 86 5227-5230 (2001)

6. Bojowald, M. Large scale effective theory for cosmological bounces. Phys. Rev. D 75 081301(R) (2007).

7. Ashtekar, A., Pawlowski, T. \& Singh, P. Quantum nature of the Big Bang. Phys. Rev. Lett. 96 141301 (2006).

8. Bojowald, M. \& Skirzewski, A. Effective equations of motion for quantum systems. Rev. Math. Phys. 18, 713-745 (2006)

9. Bojowald, M. Dynamical coherent states and physical solutions of quantum cosmological bounces. Phys. Rev. D 75, 123512 (2007).

10. Giulini, D. et al. Decoherence and the Appearance of a Classical World in Quantum Theory (Springer, Berlin, 1996).

Acknowledgements

This work was supported in part by NSF grant PHY 05-54771.

Competing financial interests

The authors declare no competing financial interests.

Reprints and permission information is available online at http://npg.nature.com/reprintsandpermissions/ 